\title{
Correction to: Extending Data for Urban Health Decision-Making: a Menu of New and Potential Neighborhood-Level Health Determinants Datasets in LMICs
}

\author{
Dana R. Thomson - Catherine Linard • Sabine Vanhuysse • Jessica E. Steele • \\ Michal Shimoni • José Siri • Waleska Teixeira Caiaffa • Megumi Rosenberg • \\ Eléonore Wolff • Taïs Grippa • Stefanos Georganos • Helen Elsey • Dana R. Thomson
}

Published online: 3 September 2019

(C) The New York Academy of Medicine 2019

Correction to: J Urban Health (2019) 96:514-536 https://doi.org/10.1007/s11524-019-00363-3

Readers should note an additional Acknowledgment for this article:

Dana Thomson is funded by the Economic and Social Research Council grant number ES/5500161/1.

The online version of the original article can be found at https://doi.org/10.1007/s11524-019-00363-3

\section{R. Thomson}

Flowminder Foundation, Stockholm, Sweden

D. R. Thomson · C. Linard · J. E. Steele

Department of Geography and Environment, University of

Southampton, Southampton, UK

D. R. Thomson $(\bowtie)$

Department of Social Statistics, University of Southampton, Southampton, UK

e-mail: dana.r.thomson@gmail.com

\section{Linard}

Spatial Epidemiology Lab, Université libre de Bruxelles (ULB), Brussels, Belgium

\section{Linard}

Department of Geography, Université de Namur, Namur, Belgium

S. Vanhuysse $\cdot$ E. Wolff $\cdot$ T. Grippa $\cdot$ S. Georganos $\cdot$

H. Elsey

Department of Geosciences, Environment and Society

(DGES-IGEAT), Université libre de Bruxelles (ULB), Brussels,

Belgium
Publisher's Note Springer Nature remains neutral with regard to jurisdictional claims in published maps and institutional affiliations.

\section{Shimoni}

Signal and Image Centre, Faculty of Electrical engineering, Royal Military Academy, Brussels, Belgium

J. Siri

International Institute for Global Health, United Nations

University, Kuala Lumpur, Malaysia

W. T. Caiaffa

Observatory for Urban Health in Belo Horizonte, School of Medicine, Federal University of Minas Gerais, Belo Horizonte, Brazil

M. Rosenberg

Center for Health Development, World Health Organization, Kobe, Japan

D. R. Thomson

Nuffield Centre for International Health and Development, University of Leeds, Leeds, UK 